\title{
Morbilidad atendida y conductas de riesgo de la niñez y adolescencia en situación de calle de Medellín, 2008
}

\author{
Morbidity and risk behavior regarding street-children in Medellin, \\ Colombia, 2008
}

Iván F. Muñoz-Echeverri, Camilo Noreña-Herrera, Beatriz E. Londoño y Carlos A. Rojas-Arbeláez

Facultad Nacional de Salud Pública, Universidad de Antioquia. Medellín, Colombia. Ivanfelipe9@gmail.com,camilonore22@gmail.com,beatriz.betthy@gmail.com, carlosrojasudea@ yahoo.com.co

Recibido 26 Octubre 2010/Enviado para Modificación 22 Marzo 2011/Aceptado 12 Abril 2011

\section{RESUMEN}

Objetivos Describir características, morbilidad atendida y conductas de riesgo para la salud de la niñez y adolescencia en situación de calle de Medellín.

Metodología Estudio observacional descriptivo y retrospectivo. Se revisaron historias clínicas de niños, niñas y adolescentes que recibieron atención médica en cinco instituciones de protección entre enero y diciembre del 2008. El análisis incluyo análisis uni y bivariado con las variables de interés. La edad fue agrupada en: niños y niñas (0-12 años) y adolescentes (13-17 años).

Resultados Se incluyeron 483 historias clínicas. El 73,1\% de los registros del sexo masculino, $73,1 \%$ adolescentes y $44,7 \%$ residían en la calle. Los principales diagnósticos fueron: Ciertas enfermedades infecciosas y parasitarias $(18,3 \%)$, factores que influyen con el estado de salud y contacto con servicios de salud (13,7 \%), enfermedades del sistema respiratorio (11,8\%). El 81,0\% consumían sustancias sicoactivas, la más consumida fue la marihuana (80,0\%). El 84,0\% reporta actividad sexual.

Conclusión La morbilidad atendida, su actividad sexual y consumo de sicoactivos evidencian una alta vulnerabilidad en salud en este grupo poblacional. Esto exige a instancias de planificación y dirección de políticas públicas en salud la garantía de su derecho constitucional a la salud a través de la ejecución de programas de promoción de la salud y la salud sexual y reproductiva y de estrategias para la prevención de enfermedades y del consumo de sicoactivos.

Palabras Clave: Jóvenes sin hogar, morbilidad, factores de riesgo, transtornos relacionados con sustancias, conducta sexual, estado de salud, Colombia (fuente: DeCS, BIREME). 


\section{ABSTRACT}

Objectives Describing the characteristics, morbidity and risk behaviour of street children from Medellin.

Methodology This was a descriptive, retrospective study. The medical charts of children aged less than 17 who sought medical attention in five protection institutions in Medellin during 2008 were reviewed. The analysis included descriptive statistics and bivariate analysis. Age was categorised into two groups: boys/girls (012 years) and adolescents (13-17 years).

Results The following characteristicswere found after reviewing 483 medical charts: being male $(73.1 \%)$, adolescent $(73.1 \%)$ and living in the streets $(44.7 \%)$. The most common diagnoses were for infectious and parasite illnesses $(21.8 \%)$, factors that affected health status and contact with health services $(13.7 \%)$, respiratory diseases $(11.8 \%)$. A total of $81.0 \%$ of all children were engaged in psychoactive substance abuse,smoking marijuana being the most common $(80.0 \%) ; 84.0 \%$ reported engaging in sexual activity.

Conclusion Infectious diseases, sexual activity and usingpsychoactive substances placed this population in a high vulnerability category. This situation demands that local health-policy makers guarantee thispopulation's constitutional right to health, through health-promotion programmes and sexual and reproductive health strategies to prevent disease and psychoactive substance abuse.

Key Words: Homeless youth, morbidity, risk factor, substance-related disorder, sexual behaviour, health status, Colombia (source: MeSH, NLM).

$\mathrm{E}$ reconocimiento de los niños, niñas y adolescentes como sujetos de derechos tiene su asiento tanto a nivel supranacional, dada la Convención Internacional Sobre los Derechos del Niño en 1989; como nacionalmente, a través del artículo 44 de la Constitución Política y, seguidamente, la Ley 1098 de 2006, o Código de la Infancia y la Adolescencia. Estos instrumentos, asimismo, comprometen al Estado y a la sociedad en general a la provisión de las condiciones necesarias para garantizarles la salud y el desarrollo humano integral $(1,2)$.

A pesar de lo dictado por estas normas, existen severas formas de violación de los derechos de la niñez y adolescencia como la situación de calle, definida como aquella población menor de 18 años que ha adoptado la calle como residencia o fuente de sobrevivencia, y que, además, se encuentra sin protección o supervisión por parte de un adulto responsable (3).

De acuerdo con autoridades gubernamentales y académicas, este fenómeno es multicausal, destacándose como sus principales factores generadores la pobreza $(4,5)$, la violencia y desintegración familiares $(6,7)$ y el fracaso y la deserción escolares $(8,9)$. Posteriormente, cuando incursionan en la calle, encuentran un 
refugio y diferentes formas de sobrevivencia como son la mendicidad (10), los trabajos informales (9) o, en otros casos, son víctimas de la explotación sexual comercial infantil o se inmiscuyen en prácticas delictivas, como el hurto y el microtráfico de drogas (3).

Según estimativos de UNICEF, este fenómeno alcanza más de 100.000 casos en América Latina (11). Para Colombia, el Instituto Colombiano de Bienestar Familiar (ICBF) anunció que para la década de los noventa, había cerca de 12000 menores de edad envueltos en esta problemática (7). Específicamente en Medellín, la cifra más reciente se registró en el año 2002, con 629 niños, niñas y adolescentes afectados (12).

La literatura internacional considera que la población víctima de esta problemática afronta notables estados de vulnerabilidad física, mental y social, propios del medio callejero (13-16). Dentro de los principales riesgos para la salud identificados están: uso y abuso de sustancias sicoactivas (17); inicio temprano de la actividad sexual y poco uso de preservativos; alto riesgo de ser víctimas de abuso y explotación sexual $(5,9)$; exposición constante a violencia y traumatismos (5); deficientes condiciones de higiene; exposición a la intemperie y a malas condiciones higiénico-sanitarias.

A pesar de esto, tanto en el nivel internacional, como nacional y local, es muy poca la información epidemiológica sobre este grupo poblacional (3), lo que limita la realización de programas de salud específicos para esta población (18).

Basados en lo anteriormente expuesto, se llevó a cabo el proyecto de investigación "Morbilidad de la Niñez en Situación de Calle, Medellín, 2008", que pretendía aportar datos estadísticos acerca de características sociodemográficas, conductas de riesgo para la salud, morbilidad y situación nutricional de la niñez y adolescencia en situación de calle de la ciudad de Medellín. Este escrito da cuenta de los resultados sobre la morbilidad atendida y las conductas de riesgo encontrados en el estudio.

\section{MÉTODOS}

Estudio observacional descriptivo y retrospectivo. Se revisaron las historias clínicas de niños, niñas y adolescentes en situación de calle, menores de 18 años, que recibieron atención en salud entre enero y diciembre de 2008 en 5 instituciones de atención a esta población, seleccionadas a conveniencia, por 
ser las más reconocidas en la ciudad en atención a la población víctima de esta problemática.

La información se recolectó en un cuestionario diseñado por los investigadores siguiendo la estructura de la historia clínica. Se incluyó información de: aspectos socio-demográficos, sintomatología y motivos de consulta, antecedentes, estado nutricional, diagnóstico y aspectos del tratamiento y la efectividad del mismo. El instrumento se sometió a prueba piloto con 30 historia clínicas. Se excluyeron 25 historias clínicas por estar duplicadas en dos instituciones diferentes.

El cuestionario fue aplicado por un médico, un profesional, cuatro estudiantes de pregrado de la Universidad de Antioquia y, además, un adolescente perteneciente a una de las instituciones. Se digitó la información en Microsoft Access 2007. Se hizo control de calidad al $100 \%$ de los cuestionarios digitados. Se realizaron procedimientos de estadística descriptiva a todas las variables. Para la mayoría de las variables se observó distribución por sexo, edad, lugar de residencia y nivel de escolaridad. Los análisis estadísticos se realizaron en el software SPSS, versión 15.

La variable edad fue agrupada teniendo en cuenta la Ley 1098 de 2006(2), así: niños y niñas (0-12 años) y adolescentes (13-17 años). La procedencia se agrupó en los municipios del Área Metropolitana del Valle de Aburrá (Medellín y los municipios circundantes) y los demás. Los diagnósticos se codificaron de acuerdo a la versión 10 de la Clasificación Internacional de Enfermedades (CIE 10) (19).

Para el desarrollo de la investigación se obtuvo consentimiento informado por escrito, firmado por cada representante de las instituciones participantes. Además, la realización de la investigación fue autorizada por escrito por el Instituto Colombiano de Bienestar Familiar, Regional Antioquia-Choco.

\section{RESULTADOS}

En las cinco instituciones de protección fueron encontradas 483 historias clínicas de atenciones en salud en el 2008. Se encontró mayor porcentaje pertenecientes al sexo masculino $(73,1 \%)$. El 73,1 \% eran adolescentes (13-17 años) al momento de la consulta. Los lugares de residencia más reportados fueron la calle $(44,7 \%)$ y las instituciones de protección $(36,4 \%)$, la residencia en hotel 
o casa fue poco reportada $(3,1 \%)$. En el 21,3\%, hubo reporte de estudios en secundaria; el 47,8 \% básica primaria; y, el 3,1 \% no tenían ningún grado de escolaridad. Respecto a la procedencia, el 49,3\% de los niños, niñas y adolescentes provenían del Área Metropolitana del Valle de Aburrá, siendo Medellín, el lugar más frecuente (45,5\%). Dentro de aquellos procedentes de fuera del Área Metropolitana, la mayor frecuencia la tuvieron el Urabá Antioqueño (18,4 \%) y al Departamento del Chocó (6,8 \%).

En 423 historias clínicas se encontró algún tipo de diagnostico. En 100 de estas, se halló el diagnóstico de niño sano $(23,6 \%$, de las historias con diagnostico). Este diagnostico se presentó con mayor frecuencia en las historias del sexo masculino, menores de 13 años, quienes residen en instituciones de protección y en los registros con reporte negativo para el consumo de psicoactivos y la actividad sexual (Tabla 1).

Tabla 1. Frecuencia (\%) del diagnostico de "niño sano" según características sociodemográficas. Medellín, 2008 ( $n=483)$

\begin{tabular}{llc}
\hline \multicolumn{1}{c}{ Variables } & Niño sano & \\
\hline \multirow{2}{*}{ Sexo } & Masculino & 26,1 \\
Grupo de Edad & Femenino & 16,5 \\
& Niños y niñas (7-12) & 35,3 \\
& Adolescentes (13-17) & 19,2 \\
Lugar de & Institución & 32,9 \\
Residencia & Casa & 20,0 \\
& Calle & 20,7 \\
& Hotel & 28,6 \\
Nivel de & Sin dato & 7,3 \\
Escolaridad & Primaria & 32,2 \\
& Secundaria & 22,4 \\
& Ninguno & 6,7 \\
Procedencia & Sin dato & 11,9 \\
& Área Metropolitana & 23,1 \\
& Otros municipios de origen & 25,8 \\
Consumo de & Sin dato & 15,2 \\
Sicoactivos & Si & 19,6 \\
& No & 33,8 \\
Reporte de & Sin dato & 29,9 \\
Actividad sexual & Si & 12,1 \\
& No & 33,3 \\
\hline
\end{tabular}


En las historias donde se encontró diagnósticos patológicos (323 historias) se identificó un total de 737 diagnósticos. Los 3 grupos de diagnósticos más frecuentes, agrupados según la CIE-10, fueron: Ciertas enfermedades infecciosas y parasitarias $(18,3 \%)$, factores que influyen con el estado de salud y contacto con los servicios de salud (13,7\%), enfermedades del sistema respiratorio $(11,8 \%)$. Se observan diferencias por sexo en la distribución de diagnósticos, ya que para el masculino las primeras causas fueron: Ciertas enfermedades infecciosas y parasitarias $(21,8 \%)$, Traumatismos $(15,3 \%)$, y enfermedades de la piel y del tejido subcutáneo (13,7 \%); mientras que para el sexo femenino: Factores que influyen en el estado de salud y contacto con los servicios de salud (27,0\%), enfermedades del sistema genitourinario $(18,9 \%)$, y enfermedades infecciosas y parasitarias $(10,7 \%)$ (Figura 1).

Figura 1. Distribución general y por sexo (\%) de los 10 primeros grupos de diagnósticos médicos, según la $\mathrm{CIE}-10$, para niños, niñas y adolescentes en situación de calle. Medellín, 2008

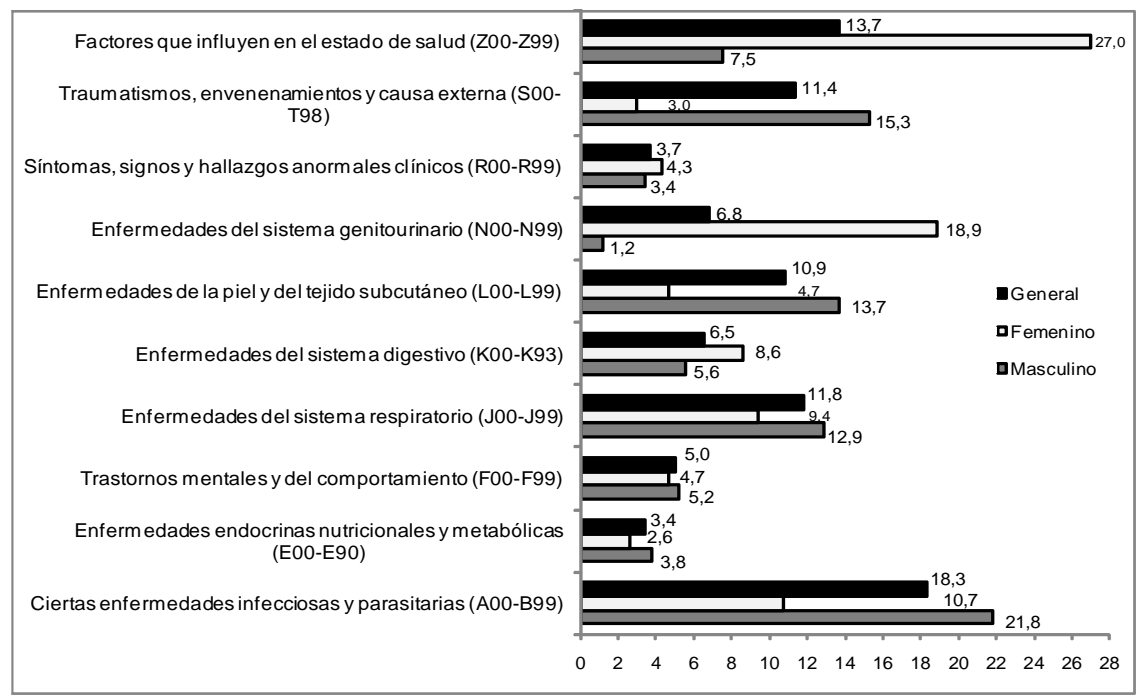

En la Figura 2 se observa que en los dos rangos de edad primaron los factores que influyen en el estado de salud (31,9\% para niños y niñas y $24,3 \%$ para adolescentes) y las enfermedades infecciosas y parasitarias (13,9\% niños y niñas 16,3\% adolescentes). Por otro lado, los diagnósticos de traumatismos y envenenamientos y del sistema genitourinario representaron las mayores diferencias de acuerdo al grupo de edad, siendo superiores en los adolescentes.

Información acerca de hábitos de consumo de psicoactivos se encontró en 404 historias clínicas, con reporte positivo de consumo en el 81,0 \%. Se encontró 
mayor consumo en el sexo femenino (95,8 \% vs. 75,5\% en el masculino), en los adolescentes $(86,0 \%)$, en quienes residían en la calle $(89,6 \%)$, en aquellos sin ningún grado escolaridad $(94,4 \%)$ y procedentes de los municipios del área metropolitana $(82,4 \%)$. En la Tabla 2 se presenta información detallada acerca del consumo en general y por tipo de sustancia, destacándose en los registros de quienes no tenían ningún grado de escolaridad, en comparación con quienes tenían algún grado escolar, mayores porcentajes de consumo de marihuana (72,2\%), inhalantes $(79,2 \%)$ y cocaína $(56,0 \%)$. En las historias con reporte de consumo de psicoactivos, se observó un promedio de consumo de 2,6 psicoactivos por persona (Desviación estándar-DE: 1,5), siendo este promedio mayor en el sexo femenino que en el masculino (3,2 y 2,3 respectivamente). El promedio de años de consumo fue 2,5 años (DE: 1,1), siendo mayor en el sexo masculino con respecto al femenino (3,0 y 2,3 años de consumo, respectivamente).

Figura 2. Distribución por edad (\%) de los 10 primeros grupos de diagnósticos médicos, para niños, niñas y adolescentes en situación de calle. Medellín, 2008

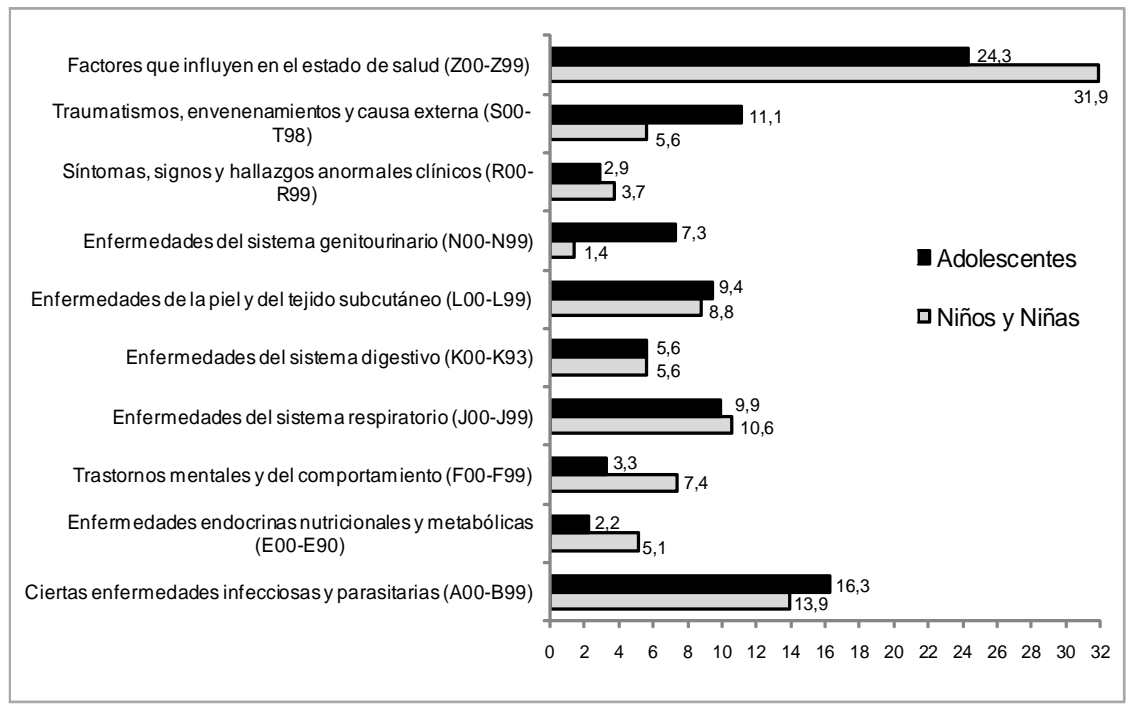

Información referente a la actividad sexual se encontró en 128 historias, en las cuales, 108 casos mencionan actividad sexual (84,0 \%). Este reporte fue mayor en el sexo femenino. En el 78,6 \% de los menores de 12 años, se encontró reporte de actividad sexual. El reporte positivo de actividad sexual sobrepasa el 90,0 \% en los residentes de hoteles $(91,7 \%)$ y consumidores de psicoactivos $(90,1 \%)$ (Tabla 3). Respecto al uso de métodos de planificación familiar, solo se obtuvo datos en 20 historias, predominando el preservativo (17 casos) y los anovulatorios (2 casos). 
Tabla 2. Reporte de consumo y tipo de psicoactivo de acuerdo a características socio demográficas. Medellín, 2008

\begin{tabular}{|c|c|c|c|c|c|c|c|c|c|}
\hline \multicolumn{10}{|c|}{ Consumo y tipo de psicoactivo } \\
\hline & \multirow{2}{*}{$\begin{array}{c}\text { Consumo } \\
\text { psicoactivos } \\
(\mathrm{n}=404) \\
\%\end{array}$} & \multirow{2}{*}{$\begin{array}{c}\text { Marihuana } \\
(\mathrm{n}=401) \\
\%\end{array}$} & \multirow{2}{*}{$\begin{array}{c}\text { Inhalantes } \\
\begin{array}{c}\text { (pegante) } \\
(n=402)\end{array} \\
\%\end{array}$} & \multirow{2}{*}{$\begin{array}{c}\text { Cocaína } \\
\text { (perico) } \\
(\mathrm{n}=401) \\
\%\end{array}$} & \multirow{2}{*}{ 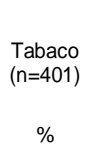 } & \multirow{2}{*}{$\begin{array}{c}\text { Benzo } \\
\text { diacepinas } \\
\text { (ruedas) } \\
(\mathrm{n}=401) \\
\%\end{array}$} & \multirow{2}{*}{$\begin{array}{c}\begin{array}{c}\text { Alcohol } \\
(\mathrm{n}=402)\end{array} \\
\%\end{array}$} & \multirow{2}{*}{$\begin{array}{c}\text { Pasta de } \\
\text { coca } \\
\text { (Basuco) } \\
(\mathrm{n}=401) \\
\%\end{array}$} & \multirow{2}{*}{$\begin{array}{c}\begin{array}{r}\text { Nitrito } \\
\text { Amilo } \\
\text { (popper } \\
(n=401) \\
\%\end{array}\end{array}$} \\
\hline & & & & & & & & & \\
\hline $\begin{array}{l}\text { Consumo } \\
\text { General }\end{array}$ & 81,0 & 65,1 & 41,0 & 36,4 & 25,9 & 17,5 & 13,2 & 8,0 & 6,2 \\
\hline \multicolumn{10}{|l|}{ Sexo } \\
\hline Masculino & 75,5 & 60,6 & 36,3 & 28,9 & 22,2 & 9,2 & 9,9 & 7,4 & 2,8 \\
\hline Femenino & 95,8 & 76,1 & 52,5 & 54,7 & 35,0 & 37,6 & 21,2 & 9,4 & 14,5 \\
\hline \multicolumn{10}{|l|}{ Grupo de Edad } \\
\hline Niños-niñas & 67,0 & 45,3 & 29,5 & 17,9 & 25,3 & 9,5 & 3,2 & 6,3 & 4,2 \\
\hline Adolescentes & 86,0 & 71,2 & 44,6 & 42,2 & 26,1 & 19,9 & 16,3 & 8,5 & 6,9 \\
\hline \multicolumn{10}{|l|}{ Residencia } \\
\hline Institución & 72,8 & 54,8 & 28,8 & 36,3 & 32,2 & 19,9 & 19,9 & 4,8 & 12,3 \\
\hline Casa & 76,9 & 69,2 & 15,4 & 7,7 & 30,8 & 7,7 & 15,4 & 0,0 & 0,0 \\
\hline Calle & 89,6 & 71,4 & 52,7 & 36,8 & 20,3 & 15,9 & 9,3 & 11,5 & 1,1 \\
\hline Hotel & 86,7 & 80,0 & 60,0 & 53,3 & 33,3 & 26,7 & 0,0 & 13,3 & 0,0 \\
\hline Sin dato & 76,1 & 66,7 & 34,8 & 37,8 & 24,4 & 15,6 & 10,9 & 4,4 & 11,1 \\
\hline \multicolumn{10}{|l|}{ Escolaridad } \\
\hline Primaria & 79,8 & 61,9 & 39,1 & 31,2 & 27,2 & 16,3 & 11,9 & 6,9 & 4,5 \\
\hline Secundaria & 85,4 & 71,6 & 39,3 & 46,6 & 28,4 & 21,6 & 20,2 & 9,1 & 13,6 \\
\hline Ninguno & 94,4 & 72,2 & 79,2 & 56,0 & 11,1 & 11,1 & 11,1 & 16,7 & 0,0 \\
\hline Sin dato & 78,7 & 64,5 & 40,9 & 34,4 & 23,7 & 17,2 & 9,7 & 7,5 & 4,3 \\
\hline \multicolumn{10}{|l|}{ Procedencia } \\
\hline Metropolitana & 82,4 & 65,0 & 43,1 & 34,0 & 25,6 & 14,8 & 13,7 & 4,4 & 7,4 \\
\hline Otros & 79,9 & 64,6 & 37,6 & 38,2 & 24,2 & 20,2 & 12,9 & 10,7 & 5,1 \\
\hline Sin dato & 85,0 & 70,0 & 50,0 & 45,0 & 45,0 & 20,0 & 10,0 & 20,0 & 5,0 \\
\hline
\end{tabular}

Tabla 3. Frecuencia de actividad sexual según características socio demográficas. Medellín, 2008 ( $n=108)$

\begin{tabular}{lll}
\hline \multicolumn{1}{c}{ Variable } & \multicolumn{1}{c}{ Reporte de Actividad Sexual } \\
\hline Sexo & Masculino & 70,0 \\
& Femenino & 93,6 \\
Grupo de Edad & Niños y niñas (7-12) & 78,6 \\
& Adolescentes (13-17) & 85,1 \\
& Institución & 78,8 \\
Lugar de & Casa & 100 \\
Residencia & Calle & 87,0 \\
& Hotel & 91,7 \\
& Sin dato & 88,9 \\
Nivel de & Primaria & 81,5 \\
Escolaridad & Secundaria & 87,0 \\
& Ninguno & 75,0 \\
& Sin dato & 92,3 \\
Procedencia & Área Metropolitana & 83,3 \\
& Otros municipios de origen & 86,2 \\
& Sin dato & 66,7 \\
Consumo de & Si & 90,1 \\
Sicoactivos & No & 27,3 \\
& Sin dato & 83,3 \\
\hline
\end{tabular}




\section{DISCUSIÓN}

De acuerdo a la revisión bibliográfica realizada, este se convierte en el primer estudio en Colombia que da cuenta de la morbilidad atendida y las conductas de riesgo de los niños, niñas y adolescentes en situación de calle, realizado a través del análisis de historias clínicas.

Los datos sobre sexo y edad que arroja este estudio, corroboran lo descrito por otros autores, que esta problemática afecta predominantemente al sexo masculino y a los adolescentes $(9,20,21)$. El bajo número de niños, niñas y adolescentes procedentes de la ciudad de Medellín, menos del $50 \%$, da cuenta de la gran movilidad, o "transhumancia", de esta población, la cual es una de sus principales características (9).

Los resultados permiten identificar los efectos de algunas condiciones del ámbito callejero en la salud de la población. Tal es el caso de la alta frecuencia de enfermedades infecciosas y parasitarias, relacionadas con condiciones de higiene corporal (22), el consumo de alimentos en mal estado $(5,23)$ o el dormir a la intemperie (13). En segundo lugar, los diagnósticos traumáticos reafirman la alta exposición a violencia en este grupo, debido a la implementación de mecanismos adaptativos y de supervivencia (10).

Igualmente, el estudio evidencia altos índices de consumo de sicoactivos y de actividad sexual, asuntos ampliamente identificados como vivencias de riesgo para la salud en este grupo poblacional $(3,6,15,17,24)$, inclusive, la prevalencia de consumo de sustancias sicoactivas encontrada fue de $81,0 \%$, cifra mayor a la prevalencia reportada para esta población en países como Brasil $(31,0 \%)$ (25), y Pakistán $(77,4 \%)(26)$.

Los hallazgos de este estudio permiten encontrar diferencias frente a los datos disponibles de morbilidad en la población general, ya que el grupo diagnóstico que encontrado en primer lugar, las enfermedades infecciosas y parasitarias, ocupa puestos secundarios para la población general de la ciudad de 5 a 14 años, de acuerdo con los indicadores básicos de Medellín, reportados en el 2006 (27). Igualmente, los factores que influyen en el contacto con los servicios de salud, que se trata fundamentalmente del consumo de psicoactivos y las conductas sexuales de riesgo, no aparecen evidenciados dentro de las 10 primeras causas de morbilidad para la población general de Medellín. Estas diferencias sugieren la existencia de inequidades en salud para esta población. 
Con relación al diagnóstico de niño, niña y adolescente sano, se destaca la mayor proporción de este hallazgo en quienes residían en instituciones de protección, lo que podría indicar que estas entidades ofrecen un impacto positivo en la salud para este grupo poblacional.

Se evidencia que la actividad sexual es mayor en el sexo femenino y, además, está práctica es acompañada del consumo de psicoactivos, situación reconocida como un factor de riesgo para la adquisición de infecciones de transmisión sexual, puesto que bajo los efectos de dichas sustancias las personas olvidan con facilidad la protección personal y son víctimas más fácilmente de la explotación sexual comercial infantil (28).

Los resultados permiten apreciar diferencias de acuerdo con el sexo, observándose como los traumatismos ocupan un importante lugar en el sexo masculino, mientras que las enfermedades relacionadas con el aparato genitourinario son relevantes en el sexo femenino. Además, se presenta una menor proporción de diagnóstico sano en el sexo femenino, a lo que se suma mayor frecuencia de consumo de psicoactivos y mayor actividad sexual. Lo anterior, puede indicar una vulnerabilidad diferencial por sexo, donde predomina la violencia física para los varones mientras que un mayor riesgo en el ámbito de la sexualidad para las niñas y las adolescentes.

Estas diferencias indican la necesidad de implementar medidas educativas y preventivas diferenciales de acuerdo al sexo, haciendo un especial énfasis en el sexo femenino. Además, los hallazgos de este trabajo llaman la atención sobre la importancia de desarrollar acciones específicas de prevención del consumo de sustancias psicoactivas y de promoción de la salud sexual y reproductiva desde edades tempranas.

Este estudio presenta limitaciones debido al trabajo con fuentes secundarias, donde no todos los registros estaban diligenciados completamente, lo que ocasionó ausencia de información por datos perdidos para algunas variables. Igualmente, dada la selección a conveniencia de las instituciones, la información reportada no puede ser extrapolable a la población general en situación de calle, ya que, por el deterioro de su situación y la alta dependencia a sustancias psicoactivas, un segmento de esta población no acude a las instituciones de protección. No obstante, se trabajo con información de las historias clínicas de las cinco instituciones con mayor volumen de atención y de mayor reconocimiento en Medellín. 
A partir de la información generada en este trabajo y, de los logros y limitaciones, se debe avanzar en la producción de evidencia epidemiológica primaria acerca de la situación de salud y morbilidad de esta población, al igual que la construcción de conocimiento acerca de estrategias preventivas en salud exitosas para ellos y ellas.

En conclusión, la morbilidad atendida, la actividad sexual y el consumo de sustancias psicoactivas en los niños, niñas y adolescentes en situación de calle de Medellín, son manifestaciones de la alta vulnerabilidad en salud de este grupo poblacional. Por lo tanto, se requiere que las instancias de planificación y dirección de las políticas públicas en salud ejecuten programas donde se realicen acciones de promoción de la salud física, sexual y reproductiva, prevención de la enfermedad y del consumo de sustancias psicoactivas, tratamiento oportuno, rehabilitación en salud y en farmacodependencia, favoreciendo la garantía y cumplimiento de su derecho constitucional a la salud. Es recomendable que estos programas prioricen la población femenina y estén dirigidos a niños y niñas desde edades tempranas y que incluya poblaciones en riesgo de caer a la situación de calle

Agradecimientos: Al comité para el desarrollo de la investigación universitaria-CODIde la Universidad de Antioquia por la financiación de este proyecto. Al Instituto Colombiano de Bienestar Familiar por la autorización para realizar el estudio. A cada una de las instituciones de protección de la ciudad de Medellín: Centro de Acogida de la Secretaría de Bienestar Social del Municipio de Medellín (operado por la Fundación Hogares Claret); Patio Don Bosco de la Fundación Ciudad Don Bosco; Patio Bosconia de la Fundación Servicio Juvenil Bosconia; y los hogares La Alegría y Miraflores de la Fundación Hogares Claret. A los encuestadores por su colaboración en la recolección de la información.

\section{REFERENCIAS}

1. Organización de Naciones Unidas para los Derechos Humanos. Convención Internacional sobre los Derechos del Niño. Ginebra, Suiza: Asamblea General de Naciones Unidas; 1989.

2. Colombia. Ley 1098 de 2006, Noviembre 8, por el cual se expide el código de la Infancia y la Adolescencia 46446. (Diario oficial 46446, de Noviembre 8; 2006).

3. Panter Brick C. Street children, human rights, and public health: a critique and future directions. Annu Rev Anthropol. 2002;31:147-71.

4. Ponce Jiménez MP. México en la calle. Alteridades. 1998;8 (15):157-64.

5. Rizzini I, Lusk MW. Children in the Streets: Latin America's Lost Generation. Child Youth Serv Rev. 1995;17(3):391-410.

6. Instituto Interamericano del Niño. Niñez en situación de calle: Un modelo de prevención de las farmacodependencias basado en los derechos humanos. 2 ed. Montevideo (Uruguay): IIN; 2002.

7. Programa de Naciones Unidas para el Desarrollo Humano PNUD. Informe sobre Desarrollo Humano 1996. Ginebra, Suiza: Ediciones Mundi-Prensa; 1996. 
8. Organización de Estados Americanos [Internet]. Disponible en: http://www.hchr.org.co/ documentoseinformes/documentos/html/informes/osi/cidh/ CIDH\%203er\%20Informe\%20Colombia\%20capitulo-13.html. Consultado Mayo 2010.

9. Galeano E, Vélez OL. La calle como forma de sobrevivencia: gaminismo, prostitución infantil y menor trabajador en el centro de Medellín. Medellín: Cámara de Comercio de Medellín; 1996.

10. Huang CC, Barreda P, Mendoza V, Guzman L, Gilbert P. A comparative analysis of abandoned street children and formerly abandoned street children in La Paz, Bolivia. Archives of Disease in Childhood. 2004;89 (9):821-6.

11. UNICEF. La niñez colombiana en cifras. Bogotá: Unicef-Oficina de Área para Colombia y Venezuela; 2002.

12. Colombia. Departamento Administrativo Nacional de Estadística. Censo sectorial de habitantes de y en la calle. informe final. Medellin: Alcaldia de Medellín, Secretaría de Solidaridad; 2003.

13. Ferguson C, McIntyre L, Kaminsky DC. Opiniones de los adultos hondureños respecto a los niños callejeros. Bol Of Sanitaria Panamericana. 1993;111 (2):105-13.

14. Akman M, Arcusin J, Pignata N, Pojomovsky J, Riterman F, Triñanes G, et al. Modalidades de abordaje médico a chicos de la calle en los hospitales públicos de la ciudad de Buenos Aires. Arch argent pediatr. 1999;97 (4):241-8.

15. Olley BO. Social and health behaviors in youth of the streets of Ibadan, Nigeria. Child Abuse \& Neglect. 2006;30 (3):271-82.

16. Ali M, de Muynck A. Illness incidence and health seeking behaviour among street children in Rawalpindi and Islamabad, Pakistan - a qualitative study. Child: Care, Health \& Development. 2005;31 (5):525-32.

17. World Health Organization. A training package on substance use, sexual and reproductive health including HIV/AIDS and STDs: Module 3, Understanding substance use among street children. Geneva: World Health Organization; 2000.

18. Faran E, Blanchard J, Zaheer HA, Reza T, Holte-McKenzie M. The HIV/AIDS Surveillance Project mapping approach: an innovative approach for mapping and size estimation for groups at a higher risk of HIV in Pakistan. AIDS. 2010;24(suppl 2):S77-S84.

19. Organización Panamericana de la Salud. Clasificación estadística internacional de enfermedades y problemas relacionados con la salud. Publicación Científica; 554. Washintong, D.C: OPS; 1995.

20. Lalor KJ. A comparative perspective. Child Abuse Negl. 1999;23(8):759-70.

21. Escuela Universitaria de Trabajo Social de Gijón. Conociendo a la infancia que vive en la calle 2004. Gijón (Asturias); 2004.

22. Centro Adentro. Habitantes de la calle: una realidad cotidiana. 2 ed. Medellín: Alcaldia de Medellín; 2006.

23. Rainer G, Britta L, Suslowati H. Height and weight as a reflection of the nutritional situation of school-aged children working and living in the streets of Jakarta. Soc Sci Med. 1996;43(4):453-8

24. Bond LS. La dolorosa realidad de los niños de la calle. Bol Oficina Sanit Panam 1993;111 (2):97-104.

25. Forster MK, Tannhauser M, Barros MT. Drug use among street children in southern Brazil,. Drug und Alcohol Dependence. 1996;43:57-62.

26. Towe VL, Hasan SU, Zafar ST, Sherman SG. Street life and drug risk behaviors associated with exchanging sex among male street children in Lahore, Pakistan. Journal of Adolescent Health 2009;44:222-8.

27. Alcaldía de Medellín. Situación de Salud en Medellín. Indicadores básicos 2005. Medellin: Alcaldía de Medellín. Secretaria de Salud; 2006.

28. Pinzón Rondón AM, Ross TW, Botero JC, Baquero Umaña MM. Prevalencia y Factores Asociados a enfermedades de transmisión sexual en menores explotados sexualmente en Bogotá, Colombia. Rev salud pública. 2009;11(3):468-79. 